\title{
UCRL-PROC-225033
}

LA W RENCE LIVERM ORE NATIONAL LABORATORY

\section{Gyrokinetic Simulations of ETG and ITG Turbulence}

A.M. Dimits, W.M. Nevins, D.E. Shumaker, G.W. Hammett, T. Dannert, F. Jenko, W. Dorland, J.N. Leboeuf, T.L. Rhodes, J. Candy, C. Estrada-Mila

October 5, 2006

21st IAEA Fusion Energy Conference Chengdu, China October 16, 2006 through October 21, 2006 
This document was prepared as an account of work sponsored by an agency of the United States Government. Neither the United States Government nor the University of California nor any of their employees, makes any warranty, express or implied, or assumes any legal liability or responsibility for the accuracy, completeness, or usefulness of any information, apparatus, product, or process disclosed, or represents that its use would not infringe privately owned rights. Reference herein to any specific commercial product, process, or service by trade name, trademark, manufacturer, or otherwise, does not necessarily constitute or imply its endorsement, recommendation, or favoring by the United States Government or the University of California. The views and opinions of authors expressed herein do not necessarily state or reflect those of the United States Government or the University of California, and shall not be used for advertising or product endorsement purposes. 


\title{
Gyrokinetic Simulations of ETG and ITG Turbulence*
}

\author{
A.M. Dimits 1), W.M. Nevins 1), D.E. Shumaker 1), G.W. Hammett 2), T. Dannert 3), F.
} Jenko 3), W. Dorland 4), J.N. Leboeuf 5), T.L. Rhodes 5), J. Candy 6), C. Estrada-Mila 7)

1) Lawrence Livermore National Laboratory, USA

2) Princeton Plasma Physics Laboratory, USA

3) Max Planck Institut fur Plasmaphysik, Germany

4) University of Maryland, USA

5) University of California, Los Angeles, USA

6) General Atomics, San Diego, USA

7) University of California, San Diego, USA

e-mail contact of main author: dimits1@llnl.gov

\begin{abstract}
Published gyrokinetic continuum-code simulations indicated levels of the electron thermal conductivity $\chi_{\mathrm{e}}$ due to electron-temperature-gradient (ETG) turbulence large enough to be significant in some tokamaks, while subsequent global particle-in-cell (PIC) simulations gave significantly lower values. We have carried out an investigation of this discrepancy. We have reproduced the key features of the aforementioned PIC simulations using the flux-tube gyrokinetic PIC code, PG3EQ, thereby eliminating global effects and as the cause of the discrepancy. We show that the late-time low-transport state in both of these sets of PIC simulations is a result of discrete particle noise, which is a numerical artifact. Thus, the low value of $\chi_{\mathrm{e}}$ along with conclusions about anomalous transport drawn from these particular PIC simulations are unjustified. In our attempts to benchmark PIC and continuum codes for ETG turbulence at the plasma parameters used above, both produce very large intermittent transport. We have therefore undertaken benchmarks at an alternate reference point, magnetic shear $s=0.1$ instead of $s=0.796$, and have found that PIC and continuum codes reproduce the same transport levels. Scans in the magnetic shear show an abrupt transition to a high- $\chi_{\mathrm{e}}$ state as the shear is increased above $s=0.4$. When nonadiabatic ions are used, this abrupt transition is absent, and $\chi_{\mathrm{e}}$ increases gradually reaching values consistent with transport analyses of DIII-D, JET, and JT60-U discharges. New results on the balances of zonal-flow driving and damping terms in late-time quasi-steady ITG turbulence and on realgeometry gyrokinetic simulations of shaped DIII-D discharges are also reported.
\end{abstract}

\section{ETG Turbulence}

Gyrokinetic continuum-code simulations of electron-temperature-gradient (ETG) turbulence have indicated a sufficiently large value of the electron thermal conductivity $\chi_{e}$ to be potentially significant in tokamaks [1]. This was cast into doubt by more recent particle-incell (PIC) simulations [2] which, using similar plasma parameters, gave significantly lower values of $\chi_{e}$. The differences between [1] and [2] were attributed to insufficient phase-space resolution and novel physics associated with global geometry [2]. We have carried out investigations [3,4] of the above discrepancy and the proposed explanations, and of the general question of whether ETG turbulence can be important in tokamaks, using gyrokinetic simulations, complemented by analytical theory.

*Work performed for US DOE at U.C. LLNL under Contract No. W7405-ENG-48, at PPPL under Contr. No. DE-AC02-76CH03073, U. Maryland under Grant No. DE-FG02-93ER54197, UCLA under Grant No. DE-FG02-04ER54740, and at General Atomics under contract Nos. DE-FG03-95ER54309 and DE-FG0292ER54141. The simulations described here made use of resources at the National Energy Research Supercomputer Center under Department of Energy Contract No. DE-AC03-76SF00098. 
Simulations were carried out (shown in Fig. 1) using the flux-tube gyrokinetic $\delta f$-PIC code, PG3EQ [4], using the same physical parameters as in [2], as well as with $r / R=0$. Both sets of simulations reproduce the key features of the results reported in [2] including (1) the first nonlinear-saturation peak in $\chi_{e}$, (2) the rise after this peak shown in Fig. 4 of Ref. [2(a)], (3) the low late-time value of $\chi_{e}\left(\chi_{e} \approx 3 \chi_{e G B}\right.$ for $r / R=0.18$ and $\chi_{e} \approx 1.5 \chi_{e G B}$ for $r / R=0$, where $\chi_{e G B}=v_{t e} \rho_{e}{ }^{2} / L_{T e}$ is the electron "gyroBohm" conductivity), and (4) the downshift in wave number of the nonlinear spectrum relative to the linear spectrum [2]. Thus, global effects are eliminated as the cause of the discrepancy between [1] and [2].

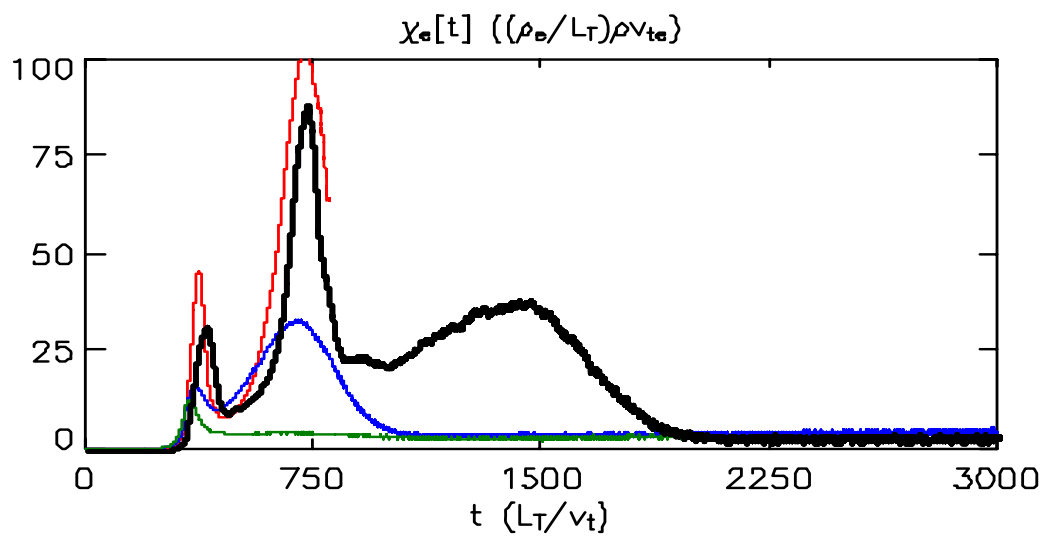

FIG. 1. $\chi_{e}$ vs. time from a particle-number and flux tube cross-section convergence study of ETG turbulence (with $r / R_{0}=0.18$ ), including runs in a flux-tube with cross-section $500 \rho_{e} \times 125 \rho_{e}$ with 2 particles/grid cell (green curve), 4 particles/grid cell (blue curve), and 16 particles/grid cell (red curve); and a flux-tube cross-section of $250 \rho_{e} \times 62.5 \rho_{e}$ with 16 particles/grid cell (black curve).

We have shown, however, that in this low- $\chi_{e}$ state in both these PG3EQ simulations and those reported in [2], discrete particle noise has become large enough to suppress the ETG turbulence [3]. Since discrete particle noise is a numerical artifact, no conclusions about the true anomalous transport level can be drawn from these simulations at late times.

The demonstration that the late-time low- $\chi_{e}$ state is a result of discrete particle noise has been facilitated by the development of a theoretical model of the interaction of $\delta f$-PIC simulation noise and physical turbulence [3]. This model reproduces the behavior of noiseaffected gyrokinetic $\delta f$-PIC simulations, both for ETG in which the noise suppresses the turbulence, and ITG where the noise and turbulence coexist and both contribute to the net transport. This model includes a detailed theoretical result for the spectrum of electrostatic potential noise fluctuations in a $\delta f$ gyrokinetic particle simulation. A comparison, shown in Fig. 2(a), of this result with the PG3EQ ETG simulations of Fig. 1 shows that the fluctuation spectra in the low- $\chi_{e}$ late-time phase of the simulations are dominated by noise.

The dominance of the noise over the turbulence in these ETG simulations occurs because the turbulence dies away, leaving behind the discrete particle noise. The small level of ExB diffusion due to noise suppresses the much larger transport due to the turbulence because the ETG turbulence is highly anisotropic in the plane perpendicular to $\boldsymbol{B}$, so that the turbulence mainly drives transport in the radial direction, while its shorter wave-length in the bi-normal direction leaves it vulnerable to noise-induced transport in this direction. 

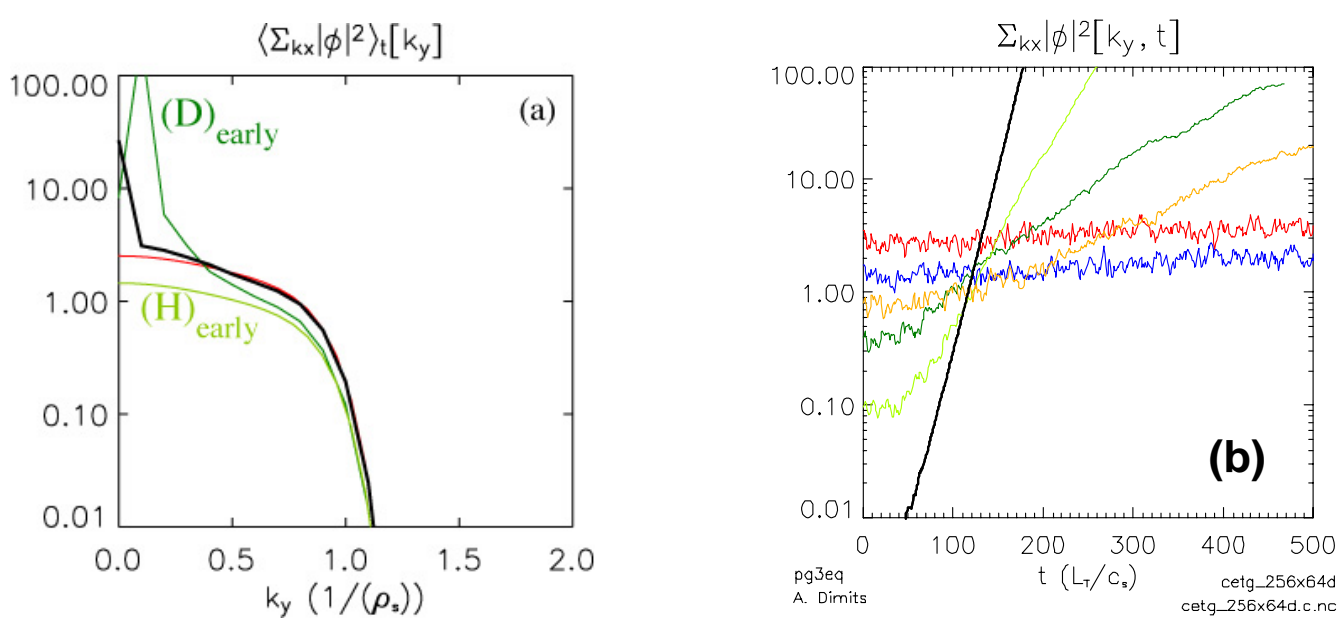

FIG. 2. (a) Fluctuation spectrum at the outboard mid-plane, averaged over the radial coordinate and time intervals $1200 L_{T} / v_{t e}<t<1300 L_{T} / v_{t e}$ (green) and $2500 L_{T} / v_{t e}<t<3000 L_{T} / v_{t e}$, (black) $v s . k_{y}$, together with the respective noise estimates (chartreuse and red). (b) Intensity of the dominant Fourier mode during the linear phase of the Bolton-Lin noise test runs. The black curve is $k_{y} \rho_{e}=0.3$ from the initial simulation (multiplied by $\left.10^{6}\right)$. In the remaining 5 curves $t$ is measured from the time of the restart. For $\left\langle w^{2}\right\rangle_{\text {initial }}=\left\langle w^{2}\right\rangle_{\text {final }}($ red $)$ and $\left\langle w^{2}\right\rangle_{\text {initial }}=(1 / 2)\left\langle w^{2}\right\rangle_{\text {final }}$ (blue) the dominant mode is $k_{y} \rho_{e}=0.1$. For $\left\langle w^{2}\right\rangle_{\text {initial }}=(1 / 4)\left\langle w^{2}\right\rangle_{\text {final }}($ gold $),\left\langle w^{2}\right\rangle_{\text {initial }}=(1 / 8)\left\langle w^{2}\right\rangle_{\text {final }}$ (green), and $\left\langle w^{2}\right\rangle_{\text {initial }}=(1 / 32)$. $\left\langle w^{2}\right\rangle_{\text {final }}$ (chartreuse) the dominant mode is $k_{y} \rho_{e}=0.2$.

We have further checked this picture of the suppression of ETG turbulence through the following "noise test" [5]. The simulation is initialized with all parameters set as they were at the beginning of the reference simulation except for the mean-squared particle weight. The initial particle weights are then chosen from a random distribution scaled such that the meansquare weight, $\left\langle w^{2}\right\rangle_{\text {initial }}$, is proportional to some multiple $\alpha$ of the late-time mean-squared particle weight from our reference simulation, $\left\langle w^{2}\right\rangle_{\text {final }}$. This test erases all memory of previous ETG turbulence other than the discrete particle noise level as quantified by the mean-squared particle weight. Figure 2(b) shows the results. The dominant mode in the initial simulation [the black curve in Fig. 2(b)] has measured growth and wavenumber in good agreement with GS2 predictions [1]. For the reinitialized simulations with $\alpha=1$ and $1 / 2$, the growth of the ETG modes is completely suppressed. For restarts initialized with progressively lower $\alpha$, the growth rate increases and approaches the low-noise result of the black curve. We conclude from this that discrete particle noise suppresses ETG turbulence transport by suppressing the linear growth of ETG modes. This is supported by further analysis, which shows that the time of maximum amplitude of each mode coincides with the time at which its linear growth rate, as modified by the noise diffusion, goes to zero.

In order to establish confidence in gyrokinetic simulation results for ETG-driven transport, a successful inter-code benchmark is highly desirable. Our attempts to benchmark PIC [4] and continuum [1] codes for ETG turbulence at the plasma parameters used in [2] produced very large, intermittent transport and strong sensitivity of the results to numerical parameters. Motivated by this and by the findings of Ref. [1], we therefore attempted benchmarks at an alternate reference point, magnetic shear $s=0.1$ instead of $s=0.796$. This change produces a large (two orders of magnitude) drop in the heat transport even though the change in the linear growth spectrum is modest. 
Figure 3 shows $\chi_{e}$ versus time from the continuum gyrokinetic codes GYRO [6], GS2 [1], and GENE [1], and from (the $\delta$ f-PIC code) PG3EQ [4]. This is the main result of this benchmark. The GYRO, GS2, and GENE simulations used similar numerical parameters. The timeinterval weighted standard deviation in $\left\langle\chi_{e}\right\rangle$ between codes yields an error in our estimate of the mean of less than $10 \%$. This agreement between codes is better than that achieved in the Cyclone ITG benchmarking exercise [7]. Additionally, it was found that the intensity of the ETG turbulent fluctuations, the mean squared ExB velocity shear, as well as the binormal and radial correlation functions from each of these codes is substantially the same.

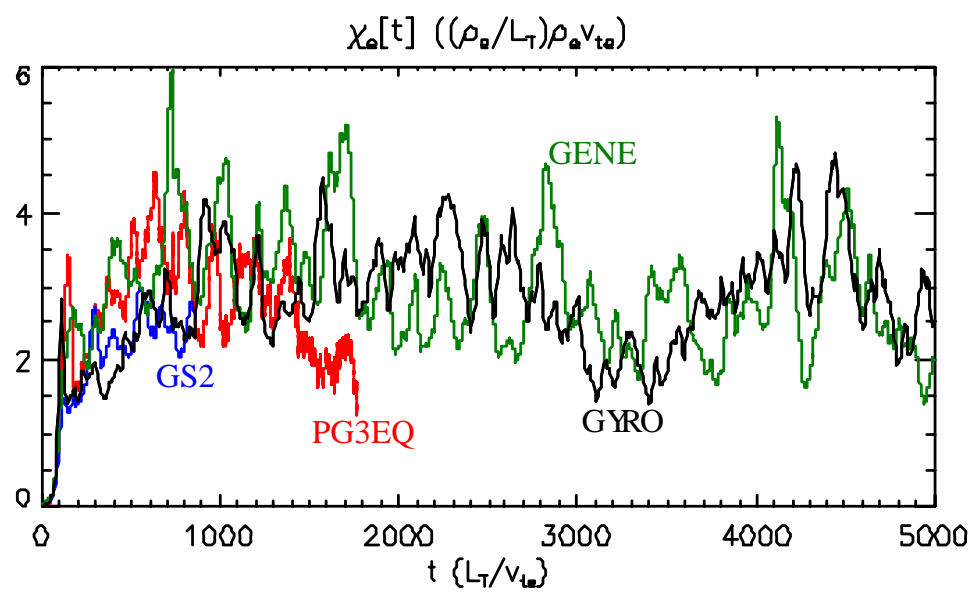

FIG. 3. $\chi_{e} \equiv-Q_{e} / n_{0} \nabla T_{0}$, in electron gyroBohm units, from GYRO (black curve), GS2 (blue curve), GENE (green curve), and PG3EQ (red curve) are plotted versus time.

In all of the simulations, the spatial system sizes were matched exactly. Convergence checks were undertaken with the GYRO, PG3EQ, and GENE codes. The GYRO case is was verified to be converged wrt. time step, velocity-space resolution, and spatial resolution in both the radial and parallel directions. The PG3EQ case was verified be converged with respect to time step and particle number. The GENE and GS2 codes have adaptive time step advances with automatic accuracy checking. A significant increase in $\chi_{e}$ with bi-normal mesh resolution has been found (and is similar) for GYRO, GENE, and PG3EQ. The binormal resolution is matched between the codes in the comparison of Fig. 3. Convergence with bi-normal resolution is being investigated further. A weak increase with binormal cross section was also found.

Scans in the magnetic shear show that as the magnetic shear is increased over the range $0.1 \leq$ $s \leq 0.35$ there is an initial large-heat-flux transient, which becomes more dramatic while the late-time $\left(t>1500 L_{T} / \mathrm{v}_{t e}\right.$ for GYRO simulations and $t>2500 L_{T} / \mathrm{v}_{t e}$ for GENE simulations) average of $\chi_{e}$ remains substantially unchanged, varying between 2.7 and $3.8\left(\rho_{e} / L_{T}\right) \rho_{e} \mathrm{v}_{t e}$. When the magnetic shear is increased further to $s=0.4$ the electron thermal conductivity takes a dramatic jump to $\left\langle\chi_{e}\right\rangle \approx 200\left(\rho_{e} / L_{T}\right) \rho_{e} \mathrm{v}_{t e}(\mathrm{GYRO})$ or $73\left(\rho_{e} / L_{T}\right) \rho_{e} \mathrm{v}_{t e}(\mathrm{GENE})$. In simulations with PG3EQ at $s=0.4$ the heat flux rises to track the results from GYRO and GENE before simulation noise suppresses the turbulence. An indication of problems with these extremely large- $\chi_{e}$ states is that the turbulent fluctuation spectrum becomes nearly monochromatic in binormal wavenumber, dominated by the lowest nonzero-wavenumber mode.

The magnetic shear scan was repeated, employing GYRO, with the adiabatic ions replaced by full gyro-kinetic ions at a mass ratio of $m_{i} / m_{e}=400$, and a somewhat larger flux-tube cross- 
section, $256 \rho_{e} \times 128 \rho_{e}$, and is shown in Fig. 4. With kinetic ions the intensity of the initial burst of turbulence increases with increasing magnetic shear. However the electron heat flux drops back down so that the late-time average remains modest, in the range of 10-14 $\chi_{e, G B}$.

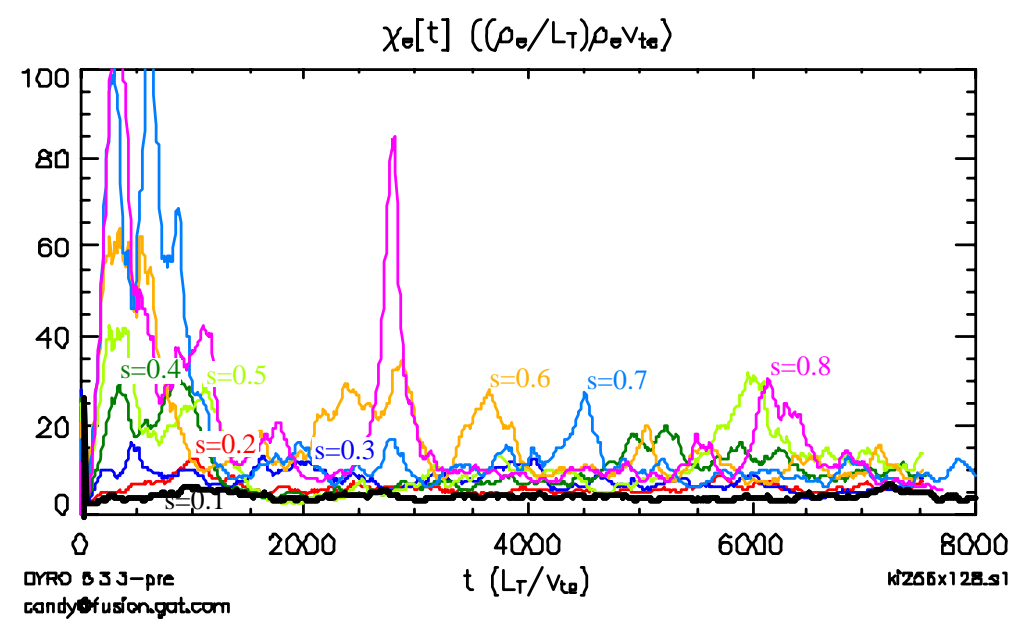

$F I G .4$. The electron thermal transport from a GYRO magnetic shear scan with kinetic ions, including $s=0.1$ (black curve), $s=0.2$ (red curve), $s=0.3$ (blue curve), $s=0.4$ (green curve), $s=0.5$ (chartreuse curve), $s=0.6$ (gold curve), $s=0.7$ (turquoise curve), and $s=0.8$ (purple curve).

Transport analyses of DIII-D [8], JET [9], and JT-60U [10] discharges suggest that ETG turbulence may be responsible for the electron heat transport across thermal barriers and in the L-mode edge of discharges with internal transport barriers. For example, in the DIII-D discharges analyzed in Ref. [9], the experimental electron heat transport normalized to $\chi_{e, G B}$ was found to be less than 1 in the internal transport barriers and of order 10 in the L-mode edge region, consistent with our ETG simulation results (with kinetic ions) at high magnetic shear. Within the internal transport barrier $\chi_{e} / \chi_{e, G B}$ is less than or of the order of one, rising to values of less than or about 25 in the L-mode plasma outside the barrier. Analysis of NSTX L-mode discharges [11] finds that $\chi_{e}$ is often substantially larger than $\chi_{i}$, and there are many cases where $\chi_{e}$ is in the range of 5-10 $\chi_{e, G B}$, consistent with our ETG simulation results.

\section{ITG turbulence: Sources and Sinks of Zonal flow.}

Anomalous ion thermal transport in some tokamak discharges [12] is believed to be caused by the toroidal ion-temperature-gradient (ITG) and related turbulence. There is now widespread evidence from simulations $[13,4]$ that zonal flows are a key component in the dynamics of such turbulence. Key to understanding the turbulence-zonal-flow system is information about the driving and dissipation of zonal-flows in the late-time nonlinear state. We have therefore developed a run-time gyrokinetic-simulation diagnostic, which has enabled us to unfold the balances of zonal-flow driving and damping terms in late-time quasi-steady ITG turbulence.

The development of this diagnostic proceeds by writing an equation (accurate to second order in gyroradius) for the rate of change of the zonal-flow $\boldsymbol{E} \boldsymbol{x B}$ shear:

$$
\frac{\partial V_{E B}^{\prime}}{\partial t} \propto\left\langle\int B d \mu d v_{\|} d \theta \frac{\partial \delta f_{g}\left(x-\rho \hat{\rho}_{x}\right)}{\partial t}\right\rangle_{f s},
$$


where $\delta f_{g}$ is the perturbed gyrocenter distribution, and \langle\rangle$_{f s}$ denotes a flux surface average. Each term in the nonlinear gyrokinetic equation yields a contribution to $\partial V_{E B}^{\prime} / \partial t$ that can be identified as a source or sink of zonal flow shear. The gyrocenter radial ExB drift flow, can be identified with the Reynolds stress. The flow associated with the difference between the gyroaveraged and gyrocenter ExB drifts can be identified as the diamagnetic Reynolds stress. Linear terms include the divergence of the perturbed $\nabla B$ and curvature-drift flows (magnetic pumping), and terms that represent the change in gyrocenter density associated with the change in energy due to $\nabla B$ and curvature drifts in the direction of the perturbed electric field. These terms are computed on the radial mesh in PG3EQ through "particle deposition" operations. To facilitate analysis, we recast this as an equation for the $\partial\left\langle\left|V_{E B}^{\prime}\right|^{2}\right\rangle_{x} / \partial t$.

Figure 5(a) shows the results of applying this diagnostic to a PG3EQ simulation using the parameters of Ref. [4]. The Reynolds stress is seen to be positive definite and is the main source of zonal flow shear. The linear terms (the sum of which is the blue curve) act as sinks.
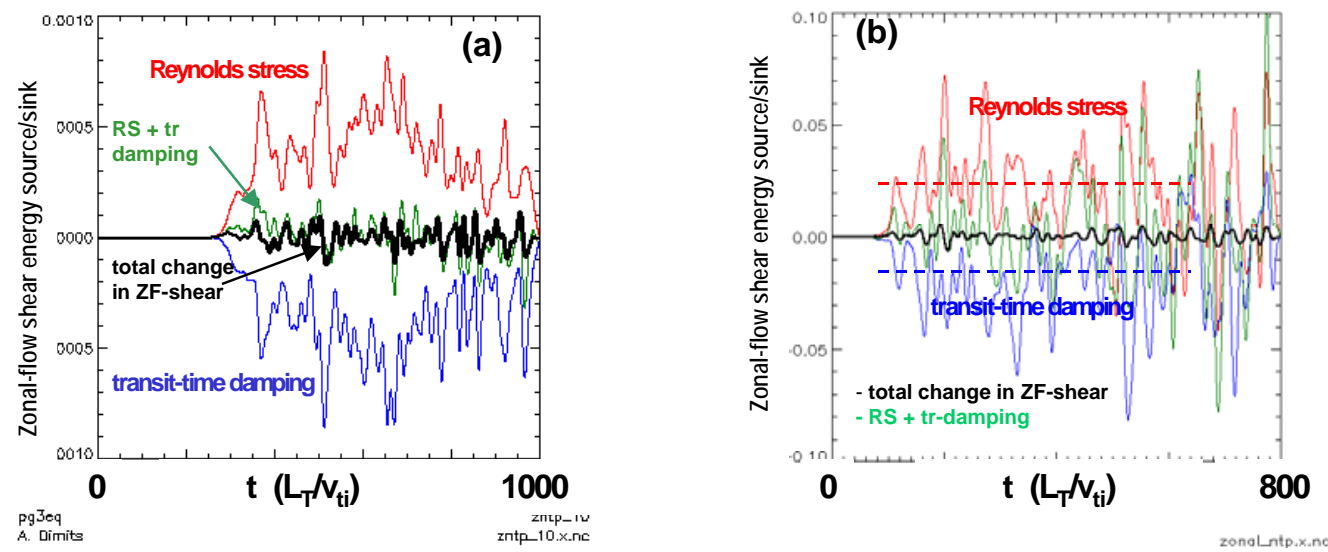

FIG. 5. Relative contributions to $\partial\left\langle\left|V_{E B}^{\prime}\right|^{2}\right\rangle_{x} / \partial t$ from a $P G 3 E Q$ simulation using the NTP base case parameters. The red curve is the Reynolds stress, the blue curve is sum of linear terms, the green curve is the sum of the Reynolds stress and linear terms, and the black curve is the total rate of change of $\left\langle\left|V_{E B}^{\prime}\right|^{2}\right\rangle_{x}$ (a) calculated over whole flux surfaces and (b) calculated by sampling at four poloidal locations (outer and inner midplane and top and bottom).

The total rate of change of $\left\langle\left|V_{E B}^{\prime}\right|^{2}\right\rangle_{x}$ is shown as the black curve, while the green curve is the sum of the Reynolds stress and linear terms. The time integral of the black curve at early time ( $\mathrm{t}=200-300)$ directly gives the initial buildup of $\left\langle\left|V_{E B}^{\prime}\right|^{2}\right\rangle_{x}$. Also, that the green curve tracks the black curve well indicates that that the Reynolds stress and linear terms capture most of the contribution. The Reynolds stress and linear damping terms are each much larger than the net rate of change of $\left\langle\left|V_{E B}^{\prime}\right|^{2}\right\rangle_{x}$. The sources and sinks can be further decomposed. The diamagnetic Reynolds stress is found to be small, and acts as a weak sink. The linear damping terms have comparable contributions from the magnetic pumping terms and the energy source terms. This diagnostic has been applied to and the same conclusions reached for a wide variety of PG3EQ simulation cases.

We have extended this analysis to provide guidance for experimental fluctuation diagnostics, which are able to measure fluctuations in a spatially localized region and are unable to 
diagnose fluctuations over an entire flux surface. It is found that 4 poloidally localized samples [see Fig. 5(b)] are sufficient to provide a resolution of the zonal-flow shear sources and sinks. Attempts made with 2 poloidally localized samples yield little useful information.

\section{ITG turbulence: Real-geometry-tokamak gyrokinetic simulations}

We have developed a real-geometry version SUMMIT/PG3EQ_NC of the gyrokinetic code PG3EQ. SUMMIT/PG3EQ_NC implements real geometry using the quasi-ballooning representation [4] of the field quantities. It has been interfaced to DIII-D equilibrium analyses from the EFIT [15], ONETWO [16], and PLOTEQ [17] codes.

Verification of PG3EQ_NC has been carried out by comparing it in detail to the earlier circular-cross-section gyrokinetic code PG3EQ [4]. Agreement to roundoff for many time steps, and across different parallel domain decompositions has been obtained when the initial loading and equilibrium are identical.

Real geometry PG3EQ_NC simulations have been undertaken using equilibria from several DIII-D shots including shot \#81499, for which a $\chi_{i}$ time history and poloidal section $\phi$ images are shown in Fig. 6, and shot \#118561. A "circularized" equilibrium with parameters taken from shot \#81499 was used as the basis for the "Cyclone" ITG turbulence simulation comparison [7]. Shot \#118561 was part of a recent DIII-D campaign in which broad wavenumber range density fluctuation measurements were performed. This range includes wavenumbers from $\sim 0.7 \mathrm{~cm}^{-1}$ to $35 \mathrm{~cm}^{-1}\left(\mathrm{k} \rho_{\mathrm{s}} \approx 0.2-10\right)$, covering the range of ITG, and ETG instabilities. Plasma instabilities were excited by using short duration neutral beam blips to modify the plasma parameters from a low-transport ohmic state. These perturbations modified the background temperature and density as well as the density fluctuation behavior.
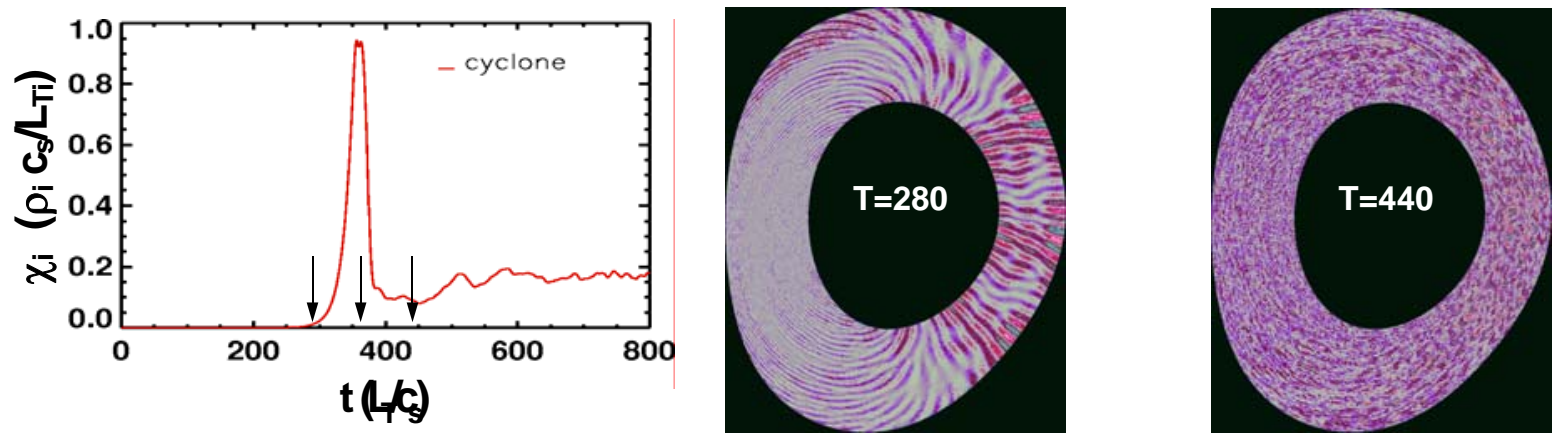

FIG. 6. Time history of $\chi_{i}$ and images of the electrostatic potential in a poloidal section from a PG3EQ_NC simulation using a real DIII-D equilibrium from EFIT and ONETWO analyses of shot $\# 081499$ at $t=4000 \mathrm{~ms}$, and $r / a=0.509$. The times in the simulation are $t=280$ and $440 L_{T i} / c_{s}$.

The measured response to these perturbations varied with wavenumber, supporting the need for broad wavenumber comparisons including both the ITG and ETG range of wavenumbers.

Figure 7 shows the maximum growth rate versus minor radius from PG3EQ_NC simulations using the equilibria from EFIT and ONETWO analyses and circularized equilibria for DIII-D shots (a) \#81499 at $\mathrm{t}=4000 \mathrm{~ms}$ and (b) \#118561 at $\mathrm{t}=1450 \mathrm{~ms}$. The shot \#81499 cases show a modest effect of shaping, with the real-geometry $\gamma(r)$ profile shifted slightly outwards relative to the circular case. The shot \#118561 cases show very little effect from shaping. Examination of the local equilibrium parameters indicates that the toroidal ITG modes become stable at 
large minor radius primarily because the magnetic shear becomes large. At small minor radius, the growth rates become small because the temperature gradient becomes small causing the dimensionless parameters $\mathrm{R} / \mathrm{L}_{\mathrm{Ti}}$ and $\eta_{\mathrm{i}}$ to fall below marginal values.
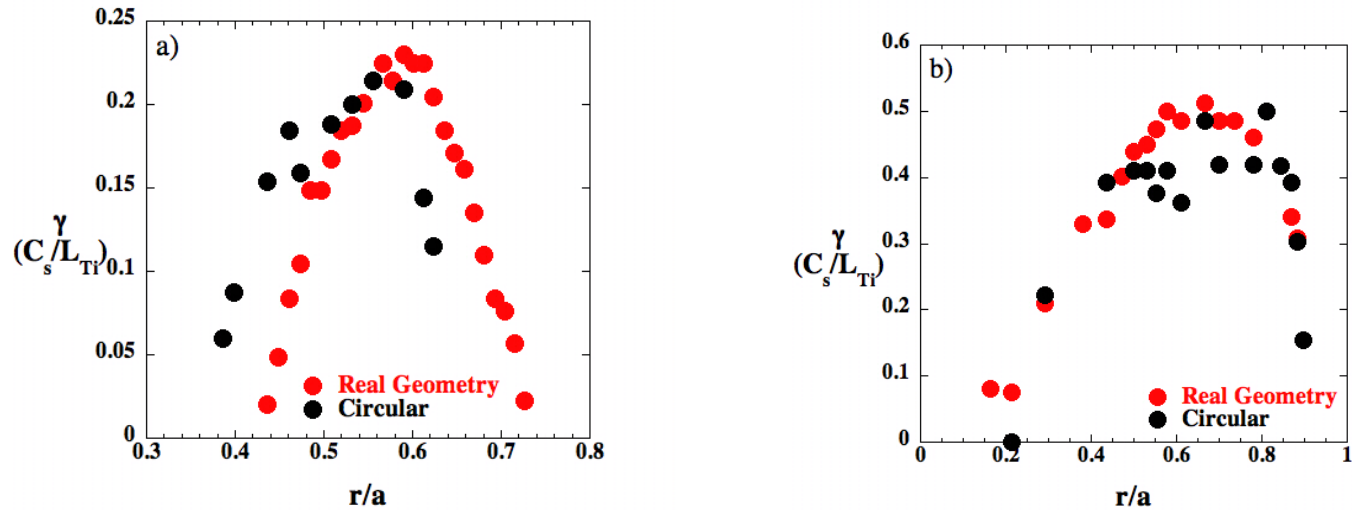

FIG. 7. Maximum ITG growth rates (in units of $c_{s} / L_{T i}$ ) vs. minor radius from $P G 3 E Q$ NC simulations using real DIII-D equilibria (red) and circularized equilibria (black) from (a) shot \#81499 at $t=4000 \mathrm{~ms}$ and (b) shot $\# 118561$ at $t=1450 \mathrm{~ms}$.

Presently being undertaken are (1) a comparison of these $\gamma(r)$ profiles with results from the GKS code [17], and (2) larger nonlinear simulation runs that will yield $\chi_{i}$ profiles.

\section{References:}

[1] JENKO, F., et al., Phys. Plasmas 7 (2000) 1905; JENKO, F. and DORLAND, W., Phys.Rev. Lett. 89 (2002) 225001.

[2] (a) LIN, Z., 2004 IAEA Mtg.; (b) LIN, Z., et al, Phys. Plasmas 12 (2005) 056125.

[3] NEVINS, W.M., et al., Phys. Plasmas 12 (2005) 122305; NEVINS, W.M., et al., Phys. Plasmas (2005) (submitted).

[4] DIMITS, A.M., et al., Phys. Rev. Letters 77 (1996), 71.

[5] BOLTON, C. and LIN, Z., private communication (April, 2005).

[6] CANDY, J. and WALTZ, R.E., J. Comput. Phys. 186 (2003) 545.

[7] DIMITS, A.M., et al., Phys. Plasmas 7, (2000) 969.

[8] STALLARD, B.W., et al., Phys. Plasmas 6, (1999) 1978.

[9] PARAIL, V.V., Nuclear Fusion 39, 429 (1999).

[10] SHIRAI, H., et al., Nuclear Fusion 39, (1999) 1713.

[11] LEBLANC, B., et al., Nuclear Fusion 44, 513 (2004).

[12] LUCE, T.C., et al., Proc.15th. Int. Conf. on Plasma Physics and Controlled Nuclear Fusion, (1995, IAEA), paper A-2-III-2.

[13] BEER, M.A., Ph.D. Thesis, (Princeton University, 1/1995).

[14] LAO, L.L., et al., Nucl. Fusion 25, (1985), 1611.

[15] PFEIFFER, W.W., et al., General Atomics Report GA-A16178 (1980), (unpublished).

[16] SAINT JOHN, H., Private Communication, (2004).

[17] KOTSCHENREUTHER, M., et al., Comp. Phys. Comm. 88 (1995) 128. 